\title{
CAVITATION EROSION INCUBATION PERIOD
}

\author{
YU-KANG ZHOU* and F. G. HAMMITT \\ Mechanical Engineering and Applied Mechanics Department, University of Michigan, Ann \\ Arbor, MI 48109 (U.S.A.)
}

(Received October 4, 1982; in revised form October 27, 1982)

\section{Summary}

To evaluate the "incubation period" (IP) stage of cavitation erosion, short-duration vibratory horn tests in tap water were made on soft aluminum alloy (aluminum alloy 1100-0) and also on a much more resistant alloy (316 stainless steel). Curves of weight loss versus time, and corresponding scanning electron microscopy photomicrographs taken during the IP, are presented and discussed. The effects of horn amplitude and temperature are investigated for "open-beaker" tests. The IP for 316 stainless steel is found to be about 500 times that for aluminum alloy 1100-O for the same amplitude and temperature. This ratio can be predicted almost exactly by applying an assumed relation between $\mathrm{MDPR}_{\max }$ and IP, i.e. $\mathrm{MDPR}_{\max }{ }^{-1}=k(\mathrm{IP})^{n}$.

Fatigue cracks and individual-blow craters were found for 316 stainless steel but only individual craters were found for aluminum alloy 1100-O, although their ductilities are approximately equal. It is found that the IP based on the eroded area only, $\mathrm{IP}_{\text {erod }}$, is much less than the conventional IP (based on the total specimen area) if IP is based on the attainment of a given mean depth of erosion MDP.

Relations between the eventual erosion rate MDPR $R_{\max }$ and the IP are considered. It is found that IP data can often be used to predict eventual $\mathrm{MDPR}_{\max }$ values according to the relation $\mathrm{MDPR}_{\max }{ }^{-1} \propto(\mathrm{IP})^{n}$ where $n \approx$ 0.93 and $n \approx 0.95$ for our vibratory and Venturi data respectively. However, different valucs for $n$ have been reported in the literature. By assuming a "characteristic" erosion-time curve the time of occurrence of $\mathrm{MDPR}_{\max }$ can also be estimated.

It is verified that only bubble collapse stresses are important in the vibratory horn test, although specimens are vibrated under very high accelerations.

*Visiting Scholar from the Shanghai Internal Combustion Engine Research Institute, Shanghai, China. 


\section{Introduction}

\subsection{General background}

Much information on cavitation erosion rates for numerous materials in various types of tests has been published over the past half-century. However, much less information is available from field devices. Also, many attempts have been made to correlate erosion rates with mechanical properties of materials [1 - 4]. In general the erosion "history" for a specimen may be divided into several "stages", e.g. the "incubation period" (IP), accelerating rate period, constant rate period, later oscillating rate periods and, in some cases, an eventual lower constant rate period $[1,2,4]$.

In any cavitation (or droplet impact) erosion test, the damage rate is generally time dependent [1-4]. Ideally (but not always) a plot of the volume loss versus time follows an S-shaped curve (Fig. 1), starting with small or zero measurable damage rate. This is called the "inception stage" or IP. After this initial period, one or several maximum rate portions occur, followed by either a continuously declining rate or alternatively a constant lower rate $[1,2,4,5]$. The exact time behavior depends on material, fluid and various other parameters of the test. The inception stage or IP is sometimes also taken to describe a damage rate stage barely measurable, i.e. a "zero-loss" condition. (Zero loss is the loss that cannot be detected by available precision balance: it is about $0.1 \mathrm{mgf}$ which is equivalent to $0.06 \mu \mathrm{m}$ for aluminum alloy $1100-\mathrm{O}$ and $0.02 \mu \mathrm{m}$ for 316 stainless steel for our test specimens of diameter $\frac{9}{16}$ in $(14.3 \mathrm{~mm})$.)

Different definitions of the IP have been used in the literature. Leith [6] defined the IP as the time interval "during which considerable plastic deformation occurs, without any apparent weight loss." He concluded that the IP depended linearly on the corrosion fatigue limit of the material. This does not appear to be generally valid today. Thiruvengadam [7] defined the IP as "that period during which the first permanent plastic dent is formed." His definition assumed that cavitation pits (or craters) are due only to fatigue effects [7]. This assumption is also not entirely valid. As recently suggested [3], the incubation period is often characterized by single-blow craters before fatigue effects become significant.

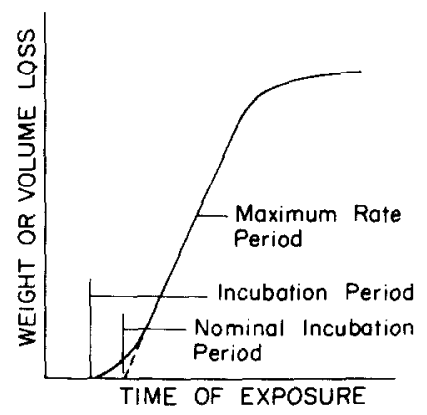

Fig. 1. Typical cavitation or liquid impact S-shaped erosion curve [1]. 


\subsection{University of Michigan practice}

For present purposes, we define the IP to be the time needed to obtain MDP $\times 10^{-3}=0.1$ in $(2.54 \mu \mathrm{m})$ (MDP, the mean depth of penetration, is defined as the volume loss divided by the exposed area). The IP has also been often defined $[1,3]$ as the time value to the intersection of the tangent from the maximum rate portion of the MDP versus time curve with the abscissa (Fig. 1). The IP is, according to the University of Michigan definition, the time to obtain a given weight loss $(\mathrm{WL})_{\mathrm{IP}}$ for a given material density. Then

$(\mathrm{WL})_{\mathrm{IP}}=(\mathrm{MDP})_{\mathrm{IP}} A \rho$

where (WL) $)_{\mathrm{IP}}$ (gf) is the weight loss for the assumed (MDP) $)_{\mathrm{IP}},(\mathrm{MDP})_{\mathrm{IP}}$ is the mean depth of penetration corresponding to the IP, i.e. $2.54 \mu \mathrm{m}$ for the University of Michigan tests, $A\left(\mathrm{~mm}^{2}\right)$ is the specimen area and $\rho\left(\mathrm{g} \mathrm{cm}^{-3}\right)$ is the specimen density. (WL) IP is then a constant for a fixed specimen diameter.

The eroded area (in the vibratory test) is always significantly [5] less than, and often only a small fraction of, the exposed (total specimen) area. In fact, because of fluid dynamic edge effects, there is always an essentially undamaged outer rim [1]. We have defined [5] an area ratio $\alpha$ :

$\alpha=\frac{\text { eroded area }}{\text { specimen area }}=\frac{A_{\text {erod }}}{A}$

$\mathrm{IP}_{\text {erod }}$ is then the time required to obtain a given (arbitrary) MDP, calculated for the actually eroded area. The conventional IP (for the total specimen area) and the IP for the eroded area only $\left(\mathrm{IP}_{\text {erod }}\right)$ are then related by eqn. (3):

$\mathrm{IP}_{\text {erod }}=\alpha \mathrm{IP}$

Then

$(\mathrm{WL})_{\mathrm{IP}_{\text {erod }}}=(\mathrm{MDP})_{\mathrm{IP}_{\text {erod }}} A_{\text {erod }} \rho$

where (WL) ${ }_{\mathrm{IP}_{\text {erod }}}$ is the weight loss for the assumed $\mathrm{MDP}_{\text {erod }}$ corresponding to $\mathrm{IP}_{\text {erod }}$, i.e. $2.54 \mu \mathrm{m}$ in the present tests. From eqns. (2) and (4),

$(\mathrm{WL})_{\mathrm{IP}_{\text {erod }}}=\alpha(\mathrm{MDP})_{\mathrm{IP}_{\text {erod }}} A \rho$

and

$\frac{(\mathrm{WL})_{\mathrm{IP}_{\text {erod }}}}{(\mathrm{WL})_{\mathrm{IP}}}=\alpha$

Obviously (WL) IP $_{\text {erod }}$ is more difficult to use than (WL) $)_{\mathrm{IP}}$ since it depends not only on the total weight loss but also on the weight loss distribution through $\alpha$. The IP for (WL $)_{\mathrm{IP}}$ erod is often much less than for (WL) $)_{\mathrm{IP}}$. However, the IP so computed should correlate better with theoretical analyses and field results.

The magnitude of the IP is closely related to the theory of dynamic indentations together with fatigue theory. It has, in general, been confirmed [8] that fatigue failure includes three stages, i.e. work hardening, crack 
formation and crack propagation. Work hardening occurs quickly and is virtually complete at an early stage in the fatigue life, probably after a few thousand stress cycles [8]. The cavitation IP is, in general, rather similar to the work-hardening stage of fatigue, i.e. before crack formation [8] becomes extensive. However, mechanisms differ somewhat between materials. Thus study of the IP is one approach to further understanding of cavitation erosion itself.

\section{Experimental results}

\subsection{Test procedure}

Easily damageable soft aluminum alloy $1100-\mathrm{O}$ and much more resistant 316 stainless steel were used for these tests to obtain a wide range of mechanical properties (Table 1). The tests were performed in the University of Michigan piezoelectric vibratory horn facility $(20 \mathrm{kHz})$. Peak-to-peak amplitudes were $1.0 \times 10^{-3}, 1.38 \times 10^{-3}$ and $1.78 \times 10^{-3}$ in $(25.4,35.1$ and $45.2 \mu \mathrm{m})$ at water temperatures of 80,160 and $200^{\circ} \mathrm{F}\left(27,71\right.$ and $\left.93^{\circ} \mathrm{C}\right)$ for 316 stainless steel, and $70^{\circ} \mathrm{F}\left(21^{\circ} \mathrm{C}\right)$ for aluminum alloy 1100-O. The suppression pressure $p_{s v}$ decreased from 1 bar with increasing temperature since these were "open-beaker" tests. $\left(p_{\mathrm{sv}}=p_{\text {vessel }}-p_{\mathrm{v}}\right.$, where $p_{\text {vessel }}$ is the static pressure in the vessel and $p_{\mathrm{v}}$ is the vapor pressure.) To obtain information during the IP as precisely as possible, test time intervals were only $10 \mathrm{~s}$ for aluminum alloy $1100-\mathrm{O}$ and $5 \mathrm{~min}$ for 316 stainless steel. However, there are still 200000 bubble collapse cycles for the aluminum alloy 1100-O IP and 6000000 for the 316 stainless steel IP. Specimens were weighed after

TABLE 1

Material mechanical properties ${ }^{a}$

\begin{tabular}{|c|c|c|c|c|c|c|}
\hline Material $^{\mathrm{b}}$ & $\begin{array}{l}U T S \\
\left(\times 10^{3} \mathrm{lbf}\right. \\
\left.\mathrm{in}^{-2}\right)\end{array}$ & $\begin{array}{l}E \\
\left(\times 10^{6} \mathrm{lbf}\right. \\
\left.\mathrm{in}^{-2}\right)\end{array}$ & $\begin{array}{l}\rho \\
\left(\mathrm{g} \mathrm{cm}^{-3}\right)\end{array}$ & $\begin{array}{l}U R \\
\left(\mathrm{lbf} \mathrm{in}^{-2}\right)\end{array}$ & $\begin{array}{l}\text { Brinell } \\
\text { hardness } \\
\text { (HB) }\end{array}$ & $\begin{array}{l}\text { Elongation } \\
(\%)\end{array}$ \\
\hline $\begin{array}{l}\text { Al alloy } \\
1100-0\end{array}$ & 11 & 10.0 & 2.71 & 6.1 & 41 & $44.5^{c}$ \\
\hline $\begin{array}{l}\text { Al alloy } \\
2024-\mathrm{T} 4\end{array}$ & 60 & 10.6 & 2.77 & 170 & 78 & $20.0^{\mathrm{c}}$ \\
\hline $\begin{array}{l}316 \text { stainless } \\
\text { steel }\end{array}$ & 81.25 & 29.0 & 7.91 & 114 & 134 & $45^{d}$ \\
\hline
\end{tabular}

a UTS, ultimate tensile strength; $E$, elastic modulus; $\rho$, density; UR $=(\text { UTS })^{2} / 2 E$, ultimat.e resilience.

${ }^{b}$ Data for Al alloys from ref. 9 unless otherwise indicated; data for 316 stainless steel from ref. 10 unless otherwise indicated.

c From ref. 11.

dFrom ref. 12. 
each test interval, observed microscopically and scanning electron microscopy (SEM) photomicrographs taken of the damaged surfaces as desired.

\subsection{Test results}

Measured data are weight loss and exposure time (Figs. 2 and 3). Figure 2 shows results for aluminum alloy $1100-0$ at room temperature and several amplitudes and Fig. 3 shows results for 316 stainless steel at several temper-

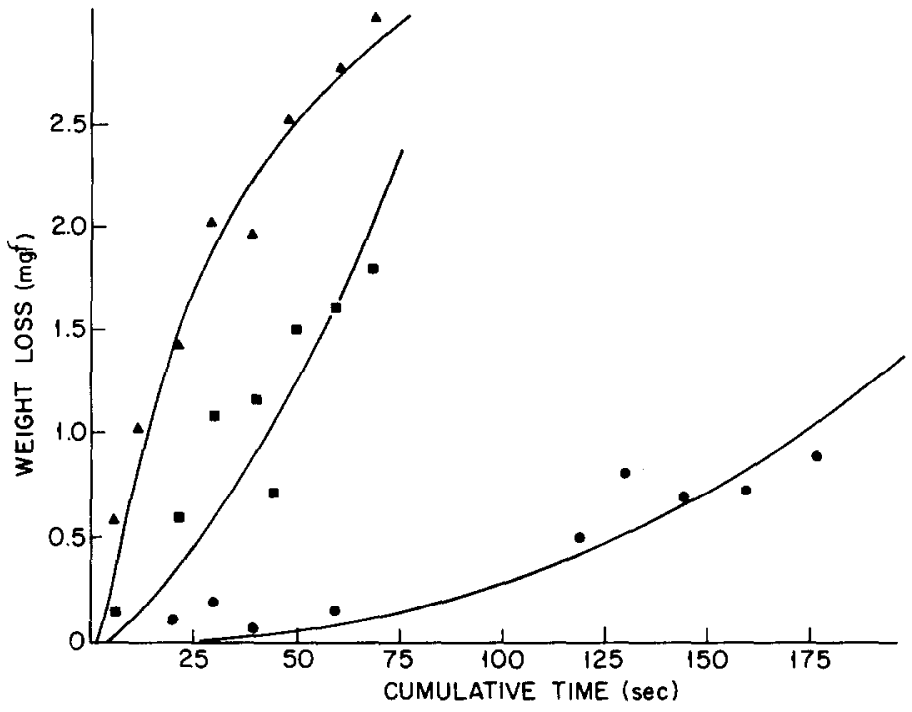

Fig. 2. Weight loss $v$ s. cumulative test time at various amplitudes for aluminum alloy 1100-O within the IP (open-beaker vibratory tests; room temperature): $\bullet, 1 \times 10^{-3}$ in; , $1.38 \times 10^{-3}$ in $; \Delta, 1.78 \times 10^{-3}$ in.

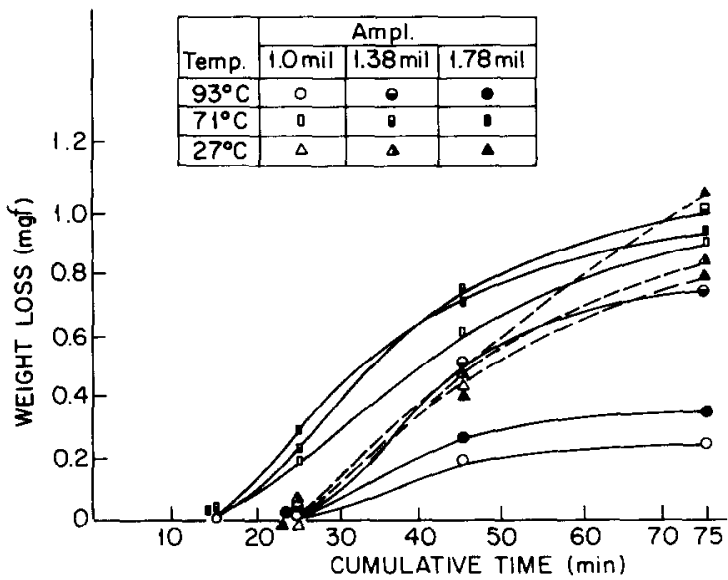

Fig. 3. Weight loss us. cumulative test time at various amplitudes and temperatures for 316 stainless steel within the IP (vibratory test). 
atures and amplitudes. All data are well within the incubation period (MDP, $1.0 \times 10^{-3}$ in). Figure 2 shows that no zero-loss period exists for aluminum alloy $1100-\mathrm{O}$ beyond about $1 \mathrm{~min}$. There are very different slopes for curves at different amplitudes for aluminum alloy $1100-\mathrm{O}$ in the inception stage, i.e. the amplitude plays a great role during that period. The zero-loss period is about $65 \mathrm{~s}$ at an amplitude of $1 \times 10^{-3}$ in and about $5 \mathrm{~s}$ at an amplitude of $1.78 \times 10^{-3}$ in. However, for 316 stainless steel (Fig. 3), there is a much greater zero-loss period. There is no weight loss within $25 \mathrm{~min}$ at $1 \times 10^{-3}$ and $1.38 \times 10^{-3}$ in or within $15 \mathrm{~min}$ at $1.78 \times 10^{-3}$ in for 316 stainless steel at all temperatures. These results can be used to estimate the amplitude exponent $n$ for these materials (see Appendix A).

Weight loss versus test time curves (Figs. 2 and 3) are essentially overall measurements of cavitation erusion. From a more basic viewpoint, the erosion results from the overall effects on adjacent material boundaries of bubble collapse. To clarify somewhat these mechanisms, a series of SEM photomicrographs were made during the inception stage (Figs. 4 - 6).

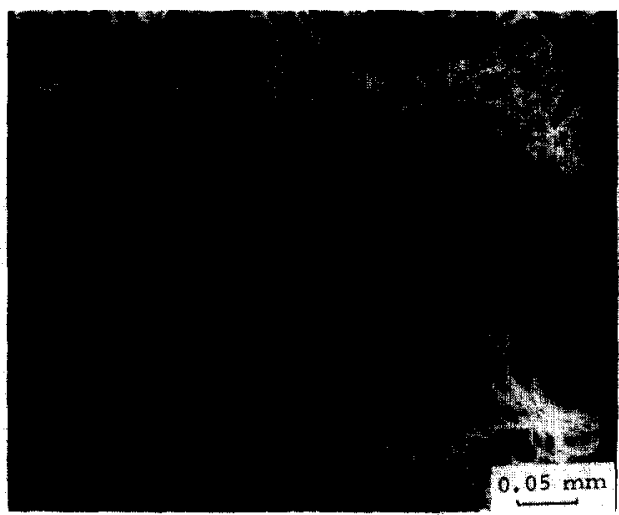

(a)

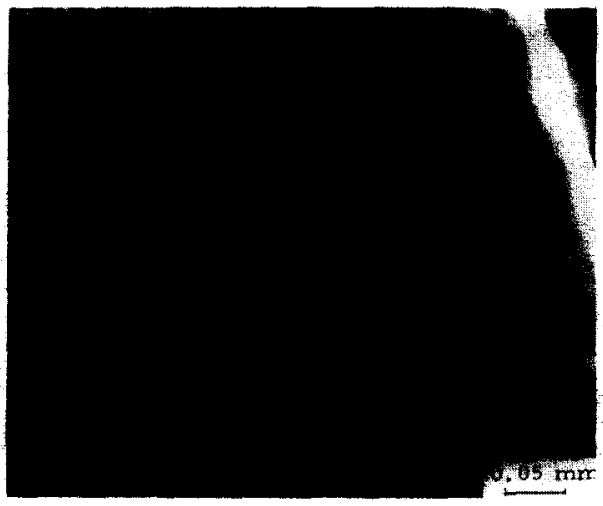

(c)

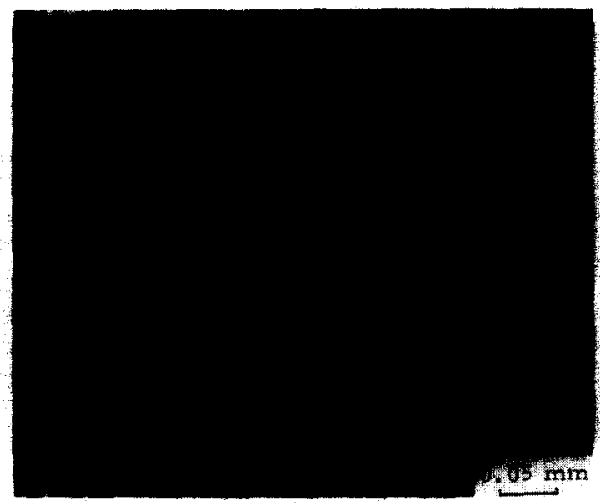

(b)

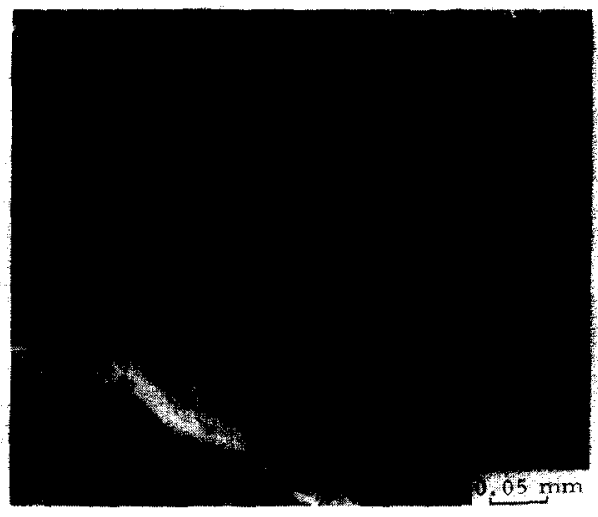

(d)

Fig. 4 (continued). 


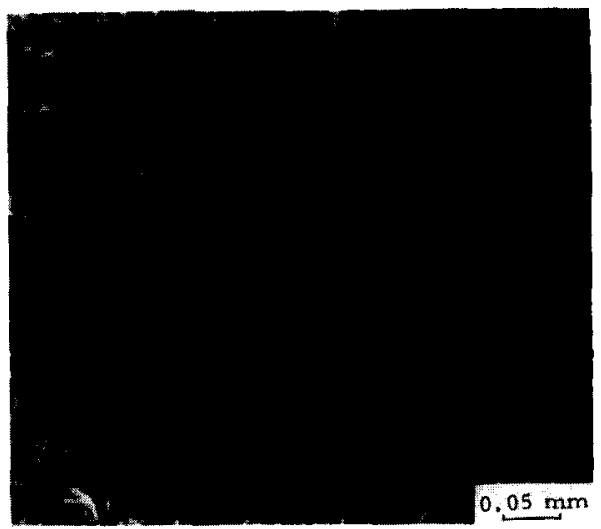

(e)

Fig. 4. Surface SEM photomicrographs in the IP (cumulative test time, $80 \mathrm{~s} ; 20 \mathrm{kHz}$ piezoelectric vibratory cavitation test; aluminum alloy $1100-0$ specimens; room temperature; atmospheric pressure): (a) central portion of the damage area (amplitude, $1.78 \mathrm{X}$ $10^{-3}$ in); (b) damage area at one-half the specimen radius (amplitude, $1.78 \times 10^{-3}$ in); (c) rim portion of the specimen (amplitude, $1.78 \times 10^{-3}$ in); (d) central portion of the damage area (amplitude, $1.0 \times 10^{-3}$ in); (e) damage area at one-half the specimen radius (amplitude, $1.0 \times 10^{-3} \mathrm{in}$ ). (Magnifications, $116 \times$.)

Figures 4(a) - 4(c) are for aluminum alloy $1100-0$ at an amplitude of $1.78 \times$ $10^{-3}$ in (the maximum used in these tests). Figures $4(\mathrm{~d})$ and $4(\mathrm{e})$ show similar SEM photomicrographs at an amplitude of $1.0 \times 10^{-3}$ in (the minimum used) for aluminum alloy 1100-O. Figure 5 shows SEM photomicrographs for various magnifications and amplitudes for aluminum alloy

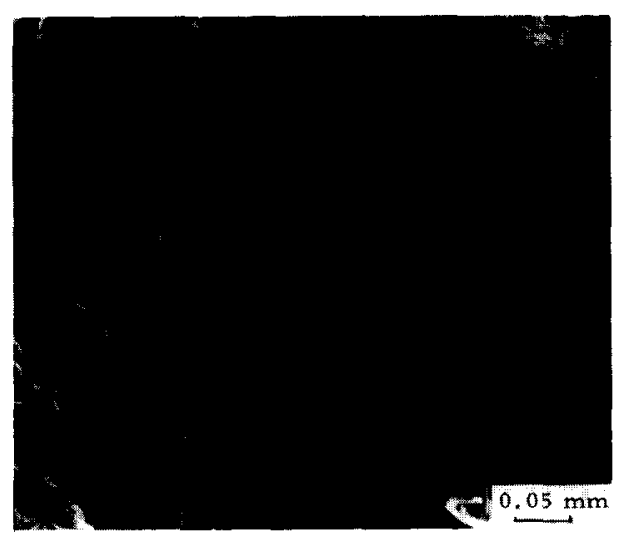

(a)

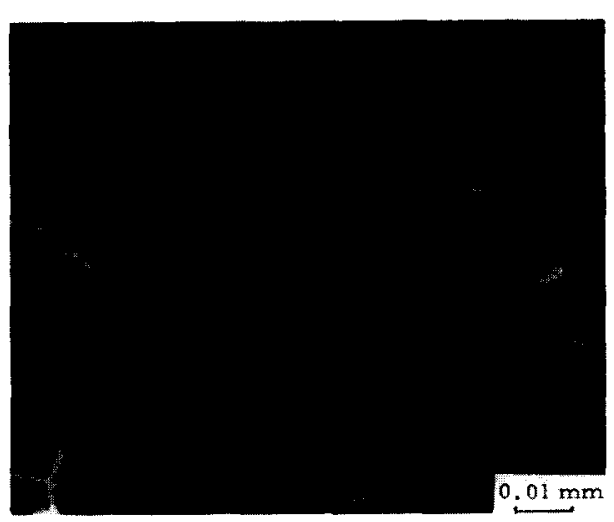

(b)

Fig. 5. Surface SEM photomicrographs taken during the IP $(20 \mathrm{kHz}$ piezoelectric vibratory cavitation test; aluminum alloy $1100-\mathrm{O}$ specimens; room temperature; atmospheric pressure): (a) central portion of the specimen (amplitude, $1.38 \times 10^{-3}$ in; total test time, $70 \mathrm{~s}$ ); (b) central portion of the specimen (amplitude, $1.0 \times 10^{-3}$ in; total test time, $60 \mathrm{~s}$ ). (Magnifications: (a) $116 \times$; (b) $580 \times$.) 


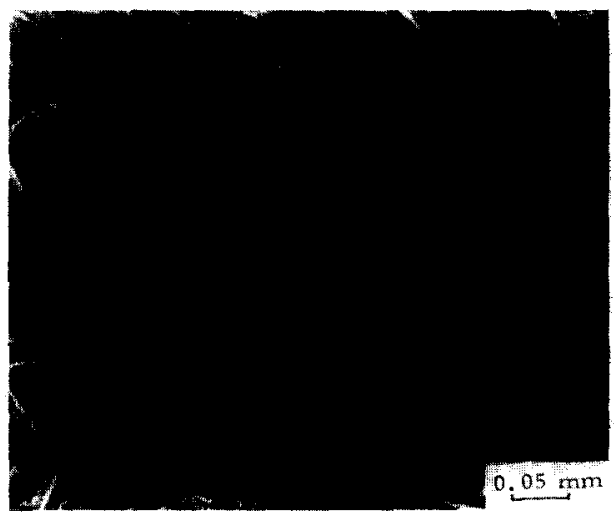

(a)

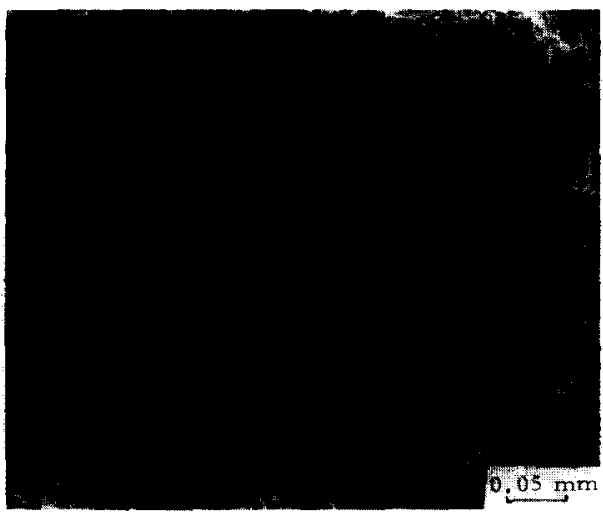

(b)

Fig. 6. Surface SEM photomicrographs in IP for 316 stainless steel (a) before testing and (b) after a cumulative test time of $75 \mathrm{~min}$ (during incubation period; $20 \mathrm{kHz}$ piezoelectric vibratory cavitation test; amplitude, $1.38 \times 10^{-3}$ in; atmospheric pressure; temperature, $80^{\circ} \mathrm{F}$ ). (Magnifications, $116 \times$.)

1100-O. Figure 6 shows SEM photomicrographs for 316 stainless steel before the test and after $75 \mathrm{~min}$ at an amplitude of $1.38 \times 10^{-3} \mathrm{in}$. Figure 7 shows weight loss versus temperature curves within the IP ( $45 \mathrm{~min}$ ) for 316 stainless steel. The maximum damage temperature is then about $140^{\circ} \mathrm{F}$ (about $60^{\circ} \mathrm{C}$ ), which is consistent with similar closed-beaker tests [5].

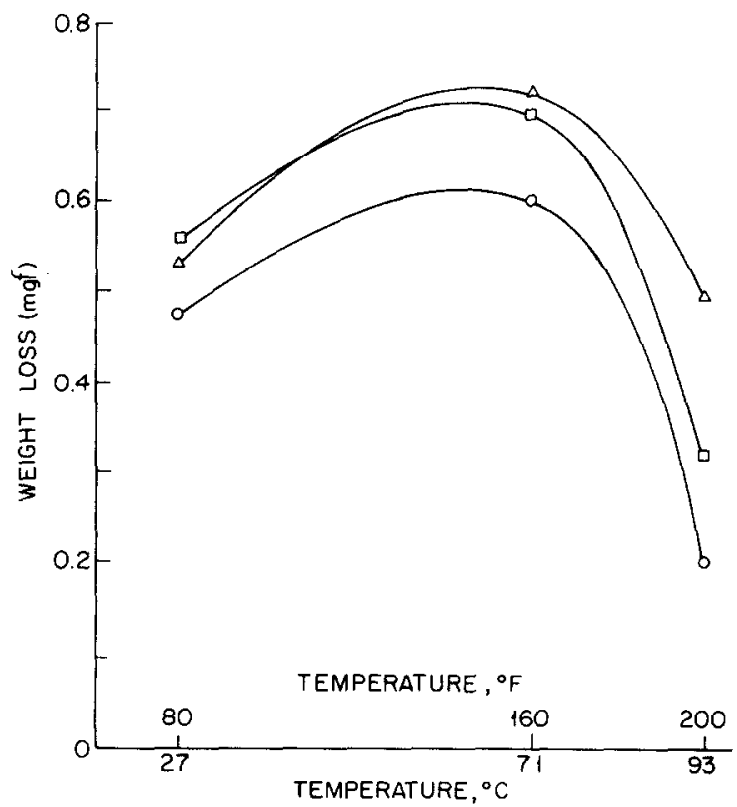

Fig. 7. Weight loss vs. test temperature at various amplitudes for 316 stainless steel within the IP (test duration, $45 \mathrm{~min}$ ): $0,1.0 \times 10^{-3}$ in; $\triangle, 1.38 \times 10^{-3}$ in $\square, 1.78 \times 10^{-3}$ in. 


\section{Discussion of results}

\subsection{Specimen surface stresses in the vibrating horn facility}

In addition to the predominant specimen surface stress $[1,4]$ due to bubble collapse (about $10^{4}-10^{5}$ bar), other stresses due to the vibration of the specimen exist, e.g. the fluid pressure oscillation itself and the stress due to the acceleration of surface particles. The first can be estimated from the "water hammer" equation:

$\Delta P_{\mathrm{wh}}=V_{\max } \rho C=2 \pi f \rho C A$

where $V_{\max }=A \omega=2 \pi f A=\pi f \times$ amplitude is the maximum velocity of the specimen surface, $\rho$ is the liquid density, $A$ is one-half the horn doubleamplitude, $\omega=2 \pi f$ is the angular velocity of vibration, $f$ is the resonant horn frequency and $C$ is the sonic velocity in the liquid. For the present study, with a maximum double-amplitude of $1.78 \times 10^{-3}$ in and a resonant frequency of $20 \mathrm{kHz}, \Delta P_{\mathrm{wh}}$ is only about 39 bar and hence cannot contribute significantly to damage.

Vibration stresses $\Delta P_{\text {vib }}$ on any grain or particle in the surface can be estimated from

$\Delta s \Delta P_{\mathrm{vib}}=\Delta F=\Delta m A \omega^{2}$

where $\Delta s$ is the surface area over which the force acts, $\Delta F$ is the force due to acceleration acting on a grain or particle and $\Delta m$ is the grain or particle mass. According to work elsewhere [13] for a double-amplitude of $25 \mu \mathrm{m}$ and a frequency of $20 \mathrm{kHz}, \Delta P_{\mathrm{vib}}=50 \mathrm{bar}$. This is not consistent with our calculation (see Appendix A), which shows that $\Delta P_{\mathrm{vib}}$ for an amplitude of $1.78 \times$ $10^{-3}$ in is only about $0.3 \mathrm{bar}$. In either case $\Delta P_{\text {vib }}$ would not contribute significantly to cavitation damage in most cases.

$\Delta P_{\text {vib }}$ stresses are not in phase with each other or with the predominant bubble collapse stress. Bubbles, of course, are nucleated during the minimum pressure part of the cycle but collapse at some undetermined time during the high pressure portion, depending on numerous other parameters of the cavitation field. Thus only bubble collapse stresses need be considered in most cases.

\subsection{Surface scanning electron microscopy photomicrographs}

The SEM photomicrographs show various damage surface configurations for the two materials during the IP. For aluminum alloy 1100-O (Fig. 4) a continuous wavy deformation structure is found. Also, a few single-blow craters occur for both of the materials. The continuous structure for aluminum alloy 1100.0 presumably results from numerous bubble collapses, most of which are not strong enough to create individual craters. In some cases, many small craters are formed within an earlier large one (Fig. 4(b)). The deformation structure is most intense, as expected, in the central portion of the specimen (Fig. 4(a)) since bubble collapses are there more numerous. The relatively undamaged specimen rim and the adjacent lightly damaged 
portion are shown in Fig. 4(c). While no fatigue cracks were found for aluminum alloy $1100-0$, they were seen for 316 stainless steel which is of the same ductility but is much stronger (Table 1). Thus the IP for aluminum alloy 1100-O appears to involve actual single-blow craters (Fig. 4(b)) rather than fatigue failures.

Figures 6(a) and 6(b) show 316 stainless steel both before the test and after exposure for $75 \mathrm{~min}$, i.e. within the IP. For this material both individual craters and fatigue cracks appear. These may then divide the surface into small separated regions which would then form larger pits, resulting in significant material removal.

Many small "microdents" (about $\left.5 \mu \mathrm{m}\left(0.2 \times 10^{-3} \mathrm{in}\right)\right)$ that are due to machining and polishing are seen in the SEM photomicrographs (Figs. 4(c) and $4(\mathrm{e})$ ). These may augment bubble nucleation, thus increasing erosion. Such small surface irregularities from any source also provide "stress raisers", which may then initiate fatigue cracks under the bubble collapse loadings. In fact, surface roughness has elsewhere been found to reduce the IP for a given material.

The weight loss curve slopes during the IP (Figs. 2 and 3) increase with horn amplitude for each material, as would be expected. They are of course much greater for the weaker material (aluminum alloy 1100-O). Thus material mechanical properties are most important, e.g. the IP of 316 stainless steel is about 500 times that for aluminum alloy $1100-\mathrm{O}$ for the same amplitude and temperature.

\subsection{Temperature effects}

The temperature of the liquid has the obvious effects on the material mechanical properties and corrosivity and thus on the IP. However, there are also "thermodynamic effects" $[1,4,5]$ which may be more important, i.e. the cavitation fluid dynamic intensity is strongly affected by the liquid temperature. Figure 7 shows the weight loss at $45 \mathrm{~min}$ versus the test temperature for 316 stainless steel (within the IP) for different amplitudes. The shapes of the open-beaker weight loss versus temperature curves for the IP are very similar to those based on $\mathrm{MDPR}_{\max }(\mathrm{Fig} .8)$ at constant $p_{\text {sv }}(2$ bar $)$, although these are for a different material (aluminum alloy 2024-T4). From Figs. 7 and 8 , the maximum damage temperature is about $140^{\circ} \mathrm{F}\left(60^{\circ} \mathrm{C}\right)$ for both tests. The decrease in the damage rate for the open-beaker test at temperatures approaching the boiling point is of course also partially a result of the decrease in the bubble collapsing pressure difference $p_{\mathrm{sv}}$ as the liquid vapor pressure and temperature increase. The maximum damage temperature is thus not substantially affected by the differences between the open-beaker and the fixed $p_{\text {sv }}$ tests, between the IP and MDPR max $_{\text {mages or between }}$ different materials (aluminum alloy 2024-T4 and 316 stainless steel).

\subsection{Relations between the IP and $M D P R_{\max }$}

The IP and MDPR $_{\max }$ are related in many cases $[1,14-16]$ by a relation of the form 


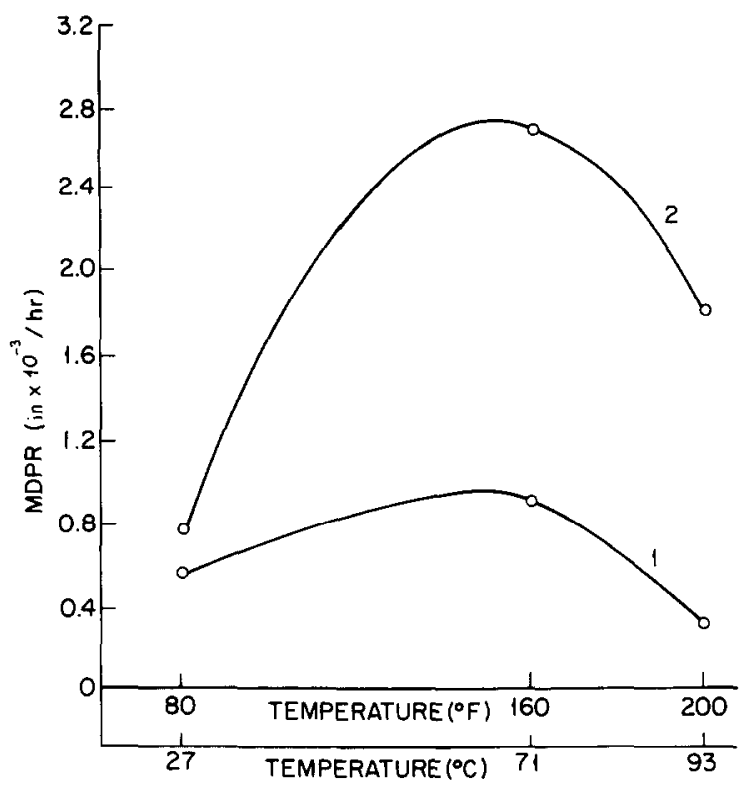

Fig. 8. MDPR vs. test temperature for aluminum alloy 2024-T4 (vibratory cavitation erosion tests; fresh water; amplitude, $1.38 \times 10^{-3}$ in ; $p_{\mathrm{sv}}=2$ bar; data from ref. 5 ): curve 1 , average MDPR based on the exposed area of the specimen; curve 2, maximum MDPR based on the exposed area of the specimen.

$\mathrm{MDPR}_{\max }{ }^{-1}=k(\mathrm{IP})^{n}$

where $k$ is an empirical coefficient which ideally is constant for all materials and tests and depends only on the detailed shape of the "characteristic" erosion curve (Fig. 1). To the extent that eqn. (9) is valid, it would be relatively easy and quick to measure the IP under prototype conditions or in a prototype machine and then to predict the remainder of the curve (Fig. 1), including MDPR $\mathrm{max}_{\max }$ and its time of occurrence [1, 14-16]. It was found $[15,16]$ that $n=0.93$ for the present vibratory tests and $n=0.95$ for the Venturi tests. Thus $n=1$ seems reasonably verified for the University of Michigan data. However, $0.7<n<1.2$ has been reported elsewhere [1]. Other pertinent semiempirical relations are as follows $[1,15,16]$ :

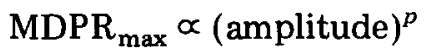

$\mathrm{MDPR}_{\max }{ }^{-1} \propto(\mathrm{UR})^{q}$

$\mathrm{MDPR}_{\max }{ }^{-1} \propto{\text { (Brinell hardness })^{r}}^{r}$

From eqn. (9) combined with eqns. (10) - (12), the IP can be related to the amplitude, the UR and the Brinell hardness. (UR $=\mathrm{UTS}^{2} / 2 E$, where UTS is the ultimate tensile strength, UR is the ultimate resilience (which is the assumed failure energy per unit volume for brittle fracture) and $E$ is the elastic modulus. Other hardness forms are equally appropriate for eqn. (12).) 
It was stated earlier that the IP for 316 stainless steel is 500 times the IP of aluminum alloy $1100-0$. The predicted value using these relations (see Appendix A) is about 500 also (actually it is equal to 513).

\section{Conclusions}

The important conclusions that can be drawn are as follows.

(1) The eventual maximum erosion rate $M_{D P R}$ max (and its time of occurrence) can be estimated from incubation period data in many cases.

(2) Fatigue cracks occur during the IP for 316 stainless steel but not for aluminum alloy $1100-0$, although their ductilities are about equal. Both materials also show individual-blow craters.

(3) The maximum damage temperature for a given duration during the incubation period for open-beaker $(1$ bar) tests is about the same as that for constant $p_{\mathrm{sv}}$ ( 2 bar) tests during the maximum damage rate period, even though different materials were compared.

(4) The IP of 316 stainless steel is about 500 times that for aluminum alloy 1100-O for the same horn amplitude and temperature. This ratio can be predicted almost exactly by applying eqn. (9): $\mathrm{MDPR}_{\max }{ }^{-1}=k(\mathrm{IP})^{n}$.

(5) Surface roughness, e.g. machining or polishing marks, may substantially affect the IP. This is confirmed by previous literature.

(6) The IP based on the eroded area only $\left(\mathrm{IP}_{\text {erod }}\right)$ is substantially less than the conventional IP (based on the total specimen area), assuming the IP to be based on the attainment of the given mean depth MDP of erosion.

\section{Acknowledgments}

Financial support was provided by National Science Foundation Grant ENG 75-2315 and internal University of Michigan funds.

\section{References}

1 F. G. Hammitt, Cavitation and Multiphase Flow Phenomena, McGraw-Hill, New York, 1980.

2 A. Thiruvendagam, Cavitation erosion, Appl. Mech. Reu., 24 (3) (1971).

3 F. G. Hammitt, M. De, J.-g. He, T. Okada and B.-H. Sun, Scale effects of cavitation including damage scale effects, Proc. 19th General Meet. of the Am. Towing Tank Conf., July 9 - 11, 1980, Ann Arbor, MI, Vol. 2, Ann Arbor Science Publishers, Ann Arbor, MI, 1981, pp. 859 - 899.

4 R. T. Knapp, J. W. Daily and F. G. Hammitt, Cavitation, McGraw-Hill, New York, 1970 ; Iowa Institute of Hydraulic Research, University of Iowa, Iowa City, IA, 1979.

5 Y.-k. Zhou and F. G. Hammitt, Vibratory cavitation tests at two bar suppression pressure, J. Chin. Shipbuild, in the press (also available as DRDA Rep. UMICH 014571. 39-I, November 1981 (Division of Research Development and Administration, University of Michigan, Ann Arbor, MI)). 
6 W. C. Leith, Cavitation damage of metals, Eng. J. (Can.), 42 (1) (1959) $43-49$.

7 A. Thiruvendagam, A unified theory of cavitation damage, J. Basic Eng., 85 (3) (1963) $365-376$.

8 P. G. Forrest, Fatigue of Metals, Pergamon, Oxford, 1962.

9 Alcoa Structural Handbook, Aluminum Company of America, Pittsburgh, PA, 1958.

10 F. G. Hammitt, C. Chao, C. L. Kling, T. M. Mitchell and D. O Rogers, ASTM round robin test with vibratory cavitation and liquid impact facilities of 6061-T6511 aluminum alloy, 316 stainless steel and commercially pure nickel, Mater. Res. Stand., 10 (10) (1970) $16-23$.

11 R. Garcia, F. G. Hammitt and R. E. Nystrom, Correlation of cavitation damage with other material and fluid properties, Erosion by Cavitation or Impingement, ASTM Spec. Tech. Publ. 408, 1966, pp. 239 - 283 (ASTM, Philadelphia, PA).

12 S. L. Hoyt (ed.), Metals Properties, McGraw-Hill, New York, 1954.

13 I. Hansson and K. A. Morch, Comparison of the initial stage of vibratory and flow cavitation erosion, Proc. 5th Int. Conf. on Erosion by Liquid and Solid Impact, Cavendish Laboratory, Cambridge, Cambs, 1979, p. 60-1.

14 F. J. Heymann, On the time dependence of the rate of erosion due to impingement or cavitation, Erosion by Cavitation or Impingement, ASTM Spec. Tech. Publ. 408, 1966, pp. 70 - 99 (ASTM, Philadelphia, PA).

15 F. G. Hammitt, Cavitation damage prediction - final report, DRDA Rep. UMICH 014456-F-1, November 1981 (Division of Research Development and Administration, University of Michigan, Ann Arbor, MI) (also published in Proc. ASME Cavitation Forum, 1983, in the press).

$16 \mathrm{~J} . \mathrm{g}$. He and F. G. Hammitt, Comparison of cavitation erosion test results from Venturi and vibratory facilities, Wear, 76 (3) (1982) $269-292$ (also available as DRDA Rep. UMICH 014456-73-I, March 1981 (Division of Research Development and Administration, University of Michigan, Ann Arbor, MI)).

\section{Appendix A}

\section{A.1. Vibratory horn parameters}

The displacement $d$, velocity $V$ and acceleration $a$ for the University of Michigan vibratory facility tests are listed in Table A1.

\section{TABLE A1}

University of Michigan vibratory horn parameters ${ }^{a}$

$\begin{array}{lc}\text { Relationships } & \\ d=A \sin (\omega t) & d_{\max }=A \\ V=A \omega \cos (\omega t) & V_{\max }=A \omega \\ a=-A \omega^{2} \sin (\omega t) & a_{\max }=A \omega^{2}\end{array}$

Values of the parameters

$A=25.4 / 2,35.0 / 2$ and $45.2 / 2 \mu \mathrm{m}$

$A \omega=1.6,2.2$ and $2.8 \mathrm{~m} \mathrm{~s}^{-1}$

$A \omega^{2}=2 \times 10^{5}, 2.7 \times 10^{5}$ and $3.6 \times 10^{5} \mathrm{~m} \mathrm{~s}^{-2}\left(\approx 2 \times 10^{4} \mathrm{~g}, \approx 2.7 \times 10^{4} \mathrm{~g}\right.$ and $\left.\approx 3.6 \times 10^{4} \mathrm{~g}\right)$

${ }^{a} \Lambda$ is onc-half of the double-horn amplitude; $g$ is the acceleration due to gravity.

\section{A.2. Vibratory horn "water hammer" pressures $\Delta P_{\mathrm{wh}}($ eqn. (7))}

From eqn. (7), $\Delta P_{\mathrm{wh}}=V_{\max } \rho C$. From Table A1, $V_{\max }=2.8 \mathrm{~m} \mathrm{~s}^{-1}, \rho=$ $1000 \mathrm{~kg} \mathrm{~m}^{-3}$ and $C \approx 1400 \mathrm{~m} \mathrm{~s}^{-1}$ for water at room temperature. Then 


$$
\begin{aligned}
\Delta P_{\mathrm{wh}} & =2.8 \mathrm{~m} \mathrm{~s}^{-1} \times 1000 \mathrm{~kg} \mathrm{~m}^{-3} \times 1400 \mathrm{~m} \mathrm{~s}^{-1} \\
& =3.92 \times 10^{6} \mathrm{~N} \mathrm{~m}^{-2} \\
& =39 \mathrm{bar}
\end{aligned}
$$

This is the maximum non-cavitating liquid pressure differential acting on the specimens. It is obviously generally negligible from the viewpoint of erosion.

\section{A.3. Surface particle acceleration stress}

From eqn. (8a),

$\Delta s \Delta P_{\text {vib }}=\Delta F=\Delta m A \omega^{2}$

So

$\Delta P_{\mathrm{vib}}=\frac{\Delta m}{\Delta s} A \omega^{2}$

$\Delta s$ is the particle surface area over which $\Delta P_{\text {vib }}$ exists. $\Delta m / \Delta s=\Delta$ (volume) $\rho_{m} /$ $\Delta s=\Delta h \rho_{\mathrm{m}}$, if we assume the particle to be a very small column, where $\Delta h$ is the effective particle height and $\rho_{\mathrm{m}}$ is the specimen density. Then

$\Delta P_{\mathrm{vib}}=\Delta h \rho_{\mathrm{m}} A \omega^{2}=\Delta h \rho_{\mathrm{m}} a$

From Table A1, the maximum acceleration $A \omega^{2}=3.6 \times 10^{5} \mathrm{~m} \mathrm{~s}^{-2}$ (for an amplitude of $1.78 \times 10^{-3}$ in). We assume that the maximum value of $\Delta h$ is $0.1 \mathrm{~mm}$. From Table $1, \rho_{\mathrm{m}}=7.91 \mathrm{~g} \mathrm{~cm}^{-3}$ for 316 stainless steel. Then

$$
\begin{aligned}
\Delta P_{\text {vib }} & =0.1 \times 10^{4} \mathrm{~m} \times 7.91 \times 10^{3} \mathrm{~kg} \mathrm{~m}^{-3} \times 3.6 \times 10^{5} \mathrm{~m} \mathrm{~s}^{-2} \\
& =2.85 \times 10^{4} \mathrm{~N} \mathrm{~m}^{-2} \\
& =0.285 \mathrm{bar}
\end{aligned}
$$

This is the maximum value of $\Delta P_{\text {vib }}$ that is acting on the specimen, and it is obviously negligible.

A.4. Ratio of incubation periods between aluminum alloy $1100-0$ and 316 stainless steel

This ratio is denoted by $\mathrm{IP}_{316} / \mathrm{IP}_{1100-0}$. According to eqn. (9),

$\mathrm{MDPR}_{\max }{ }^{-1}=k(\mathrm{IP})^{n}$

where $n=0.93$ for the University of Michigan vibratory tests. Then

$\mathrm{IP} \propto\left(\mathrm{MDPR}_{\max }{ }^{-1}\right)^{1 / n}$

and

$\frac{\mathrm{IP}_{316}}{\mathrm{IP}_{1100-\mathrm{o}}}=\left(\frac{\mathrm{MDPR}_{\max , 1100-\mathrm{o}}}{\mathrm{MDPR}_{\max , 316}}\right)^{1 / n}$ 
From our tests at $p_{\mathrm{sv}}=2 \mathrm{bar}$, an amplitude of $1.0 \times 10^{-3}$ in and $27^{\circ} \mathrm{C}$, MDPR $_{\max , 1100-\mathrm{O}}=1.0897 \times 10^{-3}{\text { in } \mathrm{h}^{-1}}^{-1}$

and

$\mathrm{MDPR}_{\max , 316}=0.00328 \times 10^{-3} \mathrm{in} \mathrm{h}^{-1}$

Also, $1 / n=1 / 0.93=1.075$, so

$\frac{\mathrm{IP}_{316}}{\mathrm{IP}_{1100-\mathrm{O}}} \approx\left(\frac{1.0897}{0.00328}\right)^{1.075}=(322.2)^{1.075}=513$

i.e. $\mathrm{IP}_{316} \approx 500 \mathrm{IP}_{1100-0}$ according to the predicting relation (eqn. (9)), which coincides almost exactly with our present measurements. Of course it is necessary to apply this procedure to other materials and test conditions to verify its actual validity in general. 